\title{
Pregnancy Medicaid Improvements in a Nonexpansion State After the Affordable Care Act
}

Jonas J. Swartz, MD, MPH ${ }^{1,2}$

Joseph Meskey, MA

Gretchen S. Stuart, MD, MPHTM ${ }^{3}$

Maria I. Rodriguez, MD, MPH

'Division of Women's Community and Population Health, Department of Obstetrics and Gynecology, Duke University, Durham, North Carolina

${ }^{2}$ Cecil G. Sheps Center for Health Services Research, University of North Carolina, Chapel Hill, North Carolina

${ }^{3}$ Division of Family Planning, Department of Obstetrics and Gynecology, University of North Carolina School of Medicine, Chapel Hill, North Carolina

${ }^{4}$ Section of Family Planning, Department of Obstetrics and Gynecology, Oregon Health \& Science University, Portland, Oregon

Conflicts of interest: authors report none

\section{CORRESPONDING AUTHOR}

Jonas J. Swartz

Duke University Medical Center

DUMC Box 3084

Durham, NC 27710

jonas.swartz@duke.edu

\begin{abstract}
One-half of women in the United States use Medicaid during pregnancy. Women living in states that did not expand Medicaid under the Patient Protection and Affordable Care Act (ACA) are at risk of losing coverage post partum. We analyzed Medicaid claims and vital statistics for the state of North Carolina for the period 2011 to 2017. North Carolina did not expand Medicaid but did alter Medicaid enrollment to meet ACA requirements. After implementation, enrollment in full Medicaid during pregnancy almost doubled, and enrollment in Medicaid for pregnant women decreased. Full Medicaid offers more comprehensive coverage and does not expire at 60 days post partum, allowing for access to crucial preventive health services including contraception and primary care.
\end{abstract}

Ann Fam Med 2021;19:38-40. https://doi.org/10.1370/afm.2615.

\section{INTRODUCTION}

$\mathrm{N}$ orth Carolina is one of 12 US states that has not expanded Medicaid eligibility under the Patient Protection and Affordable Care Act (ACA), creating a coverage gap for low-income adults. Lapses in Medicaid are particularly disruptive for the one-half of women who use Medicaid during pregnancy and who then lose medical care when their coverage expires post partum. ${ }^{1,2}$ Lack of coverage for contraception and primary care prevents women from optimizing their health for a subsequent pregnancy. ${ }^{1,2}$ Less than $40 \%$ of North Carolina Medicaid recipients return to access contraception in the first 3 months post partum. ${ }^{3}$

Enrollment processes matter because they can determine what Medicaid program women enroll in during pregnancy, and those programs differ in coverage duration and breadth. Categorical eligibility for pregnant women (Medicaid for pregnant women) extends financial eligibility to $196 \%$ of the federal poverty level in North Carolina. Coverage ends at the end of the month in which the 60th day falls post partum. In contrast, pregnant women may be categorically eligible for full Medicaid if they are disabled, have dependent children, or are aged younger than 19 years. Financial eligibility for full Medicaid varies by category but is $40 \%$ of the federal poverty level for pregnant, nondisabled adults. It also does not expire during the postpartum period. Before reform, full Medicaid included an assessment of assets (eg, real estate, bank account balances), whereas Medicaid for pregnant women did not, making enrollment in full Medicaid more financially and administratively stringent.

North Carolina did implement an electronic Medicaid enrollment system in October 2013 to meet ACA standards for simplifying Medicaid enrollment. ${ }^{1,2}$ Changes included the use of modified adjusted gross income to determine financial eligibility and shifting to a software algorithm for the assignment of categorical eligibility, thus avoiding the potential for human error. ${ }^{4}$ We aimed to determine the association of ACA-mandated changes in the enrollment process with insurance coverage for pregnancy in North Carolina. 


\section{METHODS}

We obtained medical claims and vital statistics data via the University of North Carolina Cecil G. Sheps Center for Health Services Research, the North Carolina Division of Medical Assistance, and the North Carolina State Center for Health Statistics. The data set included all women enrolled in Medicaid who were $<50$ years of age on January 1, 2011 and who had $\geq 2$ births during the period January 1, 2011 to December 31, 2017. This study was deemed exempt from review by the University of North Carolina Institutional Review Board.

We included women with $\geq 2$ Medicaid-covered births to focus on a population likely to be primarily reliant on Medicaid for health insurance coverage and less likely to access types of insurance other than Medicaid. We excluded multiple gestations, those eligible for Medicare and Medicaid, and those without coverage. We used the Medicaid member file to identify coverage type. Coverage types included (1) categorical eligibility for pregnancy (Medicaid for pregnant women), (2) categorical eligibility other than pregnancy (full Medicaid), and (3) coverage limited to emergency services and obstetric deliveries serving largely unauthorized immigrants (emergency Medicaid).

We report the proportion and number of births per month covered by each of the 3 Medicaid coverage types among women with North Carolina Medicaid coverage from 2011 to 2017. We used the Student $t$ test to compare the mean number of births by insurance type before and after policy implementation. We used Stata version 14 (StataCorp LLC) for all analyses.

\section{RESULTS}

Our sample included 172,208 pregnancies among 82,997 women (mean age: 25 years, median prior births: 1). The majority of women (48\%) had full Medicaid or Medicaid for pregnant women (43\%). A minority were covered by emergency Medicaid (9\%).

After implementation of the ACA, enrollment in full Medicaid during pregnancy increased markedly (32\% of pregnancies before enrollment reform and $58 \%$ of pregnancies after reform), whereas enrollment in Medicaid for pregnant women decreased (55\% of pregnancies before enrollment reform and $35 \%$ of pregnancies after reform) (Figure 1). Enrollment in emergency Medicaid showed a downward trend (13\% of pregnancies before enrollment reform and $7 \%$ of pregnancies after reform). The mean number of births per month covered by full Medicaid almost doubled after implementation (661 before vs 1,192 after $P_{i}$.001) (Figure 2).

\section{DISCUSSION}

Our study of North Carolina Medicaid showed a clear association between ACA-mandated reform and categorical enrollment during pregnancy, even in a state where Medicaid was not expanded. The observed shift toward better Medicaid coverage associated with ACAmandated reform might affect pregnancy and maternal health outcomes despite North Carolina's nonexpansion status. Financial eligibility for Medicaid did not expand during this timeframe; therefore, women were likely also eligible for full Medicaid before the reform.

Full Medicaid provides more comprehensive coverage; Medicaid for pregnant women covers only pregnancy-related services and expires at the end of the month in which 60 days falls post partum. This limits both interpregnancy and preconception care that is important for optimizing health. Full Medicaid allows for ongoing access to primary care, management of chronic conditions, and coverage for contraception beyond the puerperium. This is particularly important given the increasing recognition that the high prevalence of chronic medical conditions among pregnant women might be one driver of increasing maternal mortality ${ }^{1,2,6-8}$ and that obstetric clinicians should partner with primary care clinicians and specialists to optimize health before a subsequent pregnancy.

Limitations of this study include our use of administrative data and a lack of information regarding financial eligibility among enrollees. It is also unclear whether the data for North Carolina are generalizable to other states. Studies have found small changes in 


\section{Figure 2. Number of Medicaid births per month by coverage type.}

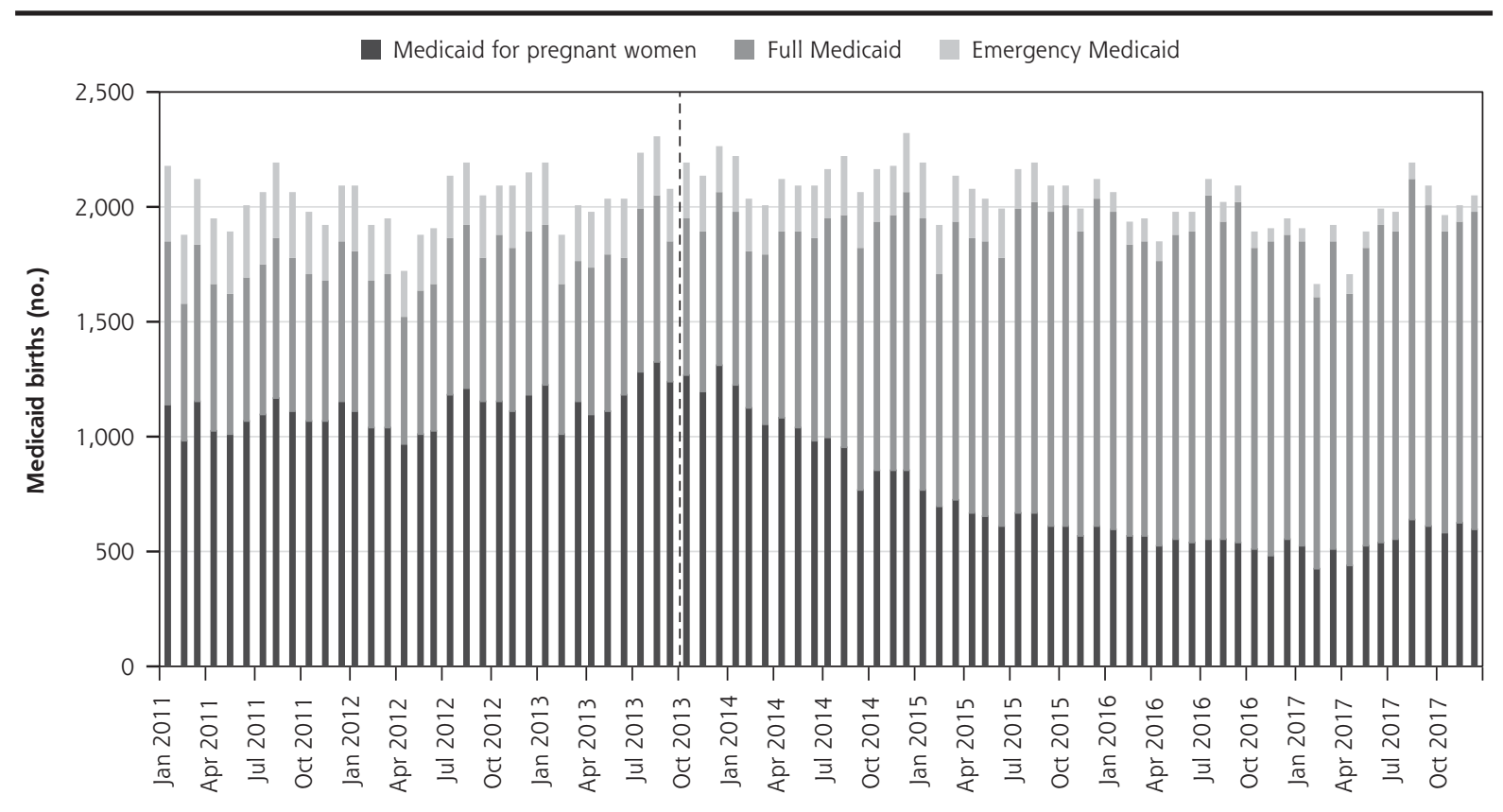

Note: The vertical dashed line denotes implementation of a software program in October 2013 that aided in Medicaid eligibility determination, in compliance with the Patient Protection and Affordable Care Act.

preterm birth rate, low birth weight, and infant mortality associated with states' Medicaid expansion status. ${ }^{9,10}$ However, our report shows that expansion status is one of multiple reform mechanisms that change Medicaid coverage. Studies investigating other state-level reforms in addition to Medicaid expansion might find previously unrecognized effects of the ACA on pregnancy outcomes. Attention to other measures of women's health might also highlight the value of access to ongoing coverage for contraception and primary care.

To read or post commentaries in response to this article, see it online at https://www.AnnFamMed.org/content/19/1/38.

Submitted February 12, 2020; submitted, revised, June 1, 2020; accepted June 9, 2020.

Key words: Patient Protection and Affordable Care Act; Affordable Care Act; ACA; Medicaid expansion; postpartum coverage; preventive health services; contraception

Funding support: Funding for this project was from the Society of Family Planning Research Fund (SFPRF18-12).

Acknowledgments: The database infrastructure used for this project was funded by the Cecil G. Sheps Center for Health Services Research; Department of Health Policy and Management, University of North Carolina Gillings School of Global Public Health; CER Strategic Initiative of University of North Carolina's Clinical and Translational Science Award (UL1TR001111); and University of North Carolina School of Medicine. The authors would like to thank Marisa E. Domino, PhD, for her help during the course of this research.

This study was deemed exempt from review by the Institutional Review Board at the University of North Carolina.

\section{References}

1. Johnson KA, Gee RE. Interpregnancy care. Semin Perinatol. 2015; 39(4):310-315.

2. Kozhimannil KB, Fontaine P. Care from family physicians reported by pregnant women in the United States. Ann Fam Med. 2013;11(4): 350-354.

3. Rutledge RI, Domino ME, Hillemeier MM, Wells R. The effect of maternity care coordination services on utilization of postpartum contraceptive services. Contraception. 2016;94(5):541-547.

4. Centers for Medicare $\&$ Medicaid Services. Targeted enrollment strategies. Accessed Nov 11, 2020. https://www.medicaid.gov/medicaid/ program-information/targeted-enrollment-strategies/index.html

5. Program Evaluation Division. Timeliness of Medicaid eligibility determinations declined due to challenges imposed by NC FAST and Affordable Care Act implementation. Final report to the Joint Legislative Program Evaluation Oversight Committee. Report Number 2016-04. Published online Apr 11, 2016. Accessed Nov 11, 2020. https://www.ncleg.net/PED/Reports/documents/Medicaid Eligibility/Medicaid_Eligibility_Final_Report.pdf

6. Chatterjee S, Kotelchuck M, Sambamoorthi U. Prevalence of chronic illness in pregnancy, access to care, and health care costs: implications for interconception care. Womens Health Issues. 2008;18(6 Suppl):S107-S116.

7. American College of Obstetricians and Gynecologists. ACOG Committee Opinion No. 736: optimizing postpartum care. Obstet Gynecol. 2018;131(5):e140-e150.

8. Koshar JH, Lee KA, Goss G, Heilemann MS, Stinson J. The Hispanic teen mother's origin of birth, use of prenatal care, and maternal and neonatal complications. J Pediatr Nurs. 1998;13(3):151-157.

9. Brown CC, Moore JE, Felix HC, et al. Association of state Medicaid expansion status with low birth weight and preterm birth. JAMA. 2019;321(16):1598-1609.

10. Bhatt $C B$, Beck-Sagué $C M$. Medicaid expansion and infant mortality in the United States. Am J Public Health. 2018;108(4):565-567. 